\title{
Editorial
}

\section{Aprender en carreras de formación de profesionales de la salud: del novato al experto}

Elaborar una editorial que reflexione sobre el aprendizaje en el campo de las ciencias de la salud implica desandar un camino construido en los últimos años a partir de las funciones específicas como asesora pedagógica en la Facultad de Medicina y de las lecturas realizadas en el trayecto profesional como investigadora del campo educativo. Camino que vuelve a recorrerse para ubicar lo trabajado en contexto: estamos hablando de aprendizaje adulto en el marco de determinadas carreras de formación profesional.

En tal sentido, nos planteamos, a modo de guía, algunos interrogantes que consideramos van a orientar el texto reflexivo que se está pretendiendo:

Para abrir la reflexión quisiéramos preguntarnos sobre ¿qué significa aprender? Cómo son estos procesos? y ¿cuáles son las metas en los procesos de aprendizaje profesional?

\section{Entonces,}

\section{¿Qué significa aprender? y ¿cómo son estos procesos?}

Todas las investigaciones actuales entienden al aprendizaje humano como un proceso vinculado a la cognición. Esto significa que "aprender" algo implicaría tiempo y de ninguna manera sería una cualidad automática o instantánea, sino procesual. En este sentido, el proceso de "aprender" tendría características comunes entre los individuos que compartimos la especie, pero también particularidades inherentes a cada uno, y por ello, sería conveniente referirse a "Ios procesos de aprendizaje", señalando con el plural sus particularidades. De esta manera, los sujetos aprenderíamos a partir de cualidades comunes que nos vinculan como especie, pero con singularidades propias de la etapa evolutiva en la que nos encontremos, del contexto al cual pertenezcamos: pertenencia social y cultural, entre otros.

Considerando estas dos premisas: "aprender es una cualidad procesual" y "aprendemos de manera singular" es que reflexionamos sobre la necesidad de considerar los aspectos temporales e idiosincráticos al momento de emprender cualquier acción que pretenda aprendizajes. En este sentido, se ha estudiado sobre los tiempos que demandan construir competencias profesionales y sobre la necesidad permanente de tener identificados los conocimientos previos que nos "habitan" (aspectos vinculados a lo idiosincrático), para desde esos conocimientos previos podamos modificar o complejizar nuestros aprendizajes y prácticas.

Vinculado a lo anterior, y planteado de modo implícito párrafos arriba, queremos señalar que los procesos de aprendizaje implicarían construcciones activas de los sujetos que los llevan adelante. Quien realmente aprende, lo hace desde la disposición y la acción sobre "eso" que pretende aprender. $Y$ cuando decimos "acción sobre" nos referimos precisamente a los procesos de construcción que como individuos realizamos sobre los objetos de aprendizaje. Procesos de construcción que, desde nuestro posicionamiento ontológico y epistemológico, llegan en un momento a desdibujar la conocida división "sujeto-objeto". Consideramos que este nuevo dibujo en el que no se podría diferenciar claramente lo que se aprendió de quien lo aprendió, ya que ambas partes se han modificado mutuamente, es la clave para comprender lo que se denomina como "aprendizaje profundo", en el que el sujeto otorga (y a la vez 
descubre), significados alrededor del conocimiento. Proceso en el que intervienen conocimientos construidos por otros, pero que son de-construidos por el propio pensamiento, y en este sentido: conocimiento y pensamiento se vuelven uno en aquel que aprende. Como podrán aseverar, esta fusión no siempre se logra y al no lograrse genera sólo conocimientos frágiles, inertes, ingenuos, rituales, que siempre corren el riesgo de ser olvidados.

Si bien, durante el escrito hemos hecho hincapié en procesos individuales, y entendemos que aprender es un proceso psicológico-cognitivo (siendo el individuo el protagonista principal de la mayoría de las teorizaciones al respecto), no quisiéramos dejar de mencionar que también hay valiosos avances en materia de psicología cognitiva que ponen el acento en los aspectos colectivos de la cognición.

Como mencionábamos párrafos arriba, aprendemos en y desde diferentes contextos que nos atraviesan: individuales, familiares, profesionales, sociales, culturales, y al atravesarnos nos "condicionan", nos "transforman", nos "habitan", nos asemeja a otros y también nos diferencia, convirtiéndose en aspectos ineludibles al momento de construir los aprendizajes. Dichos contextos además de "habitarnos" son también continentes de multiplicidad de conocimientos que se distribuyen infinitamente en personas y objetos culturales. Los procesos de cognición humana son también procesos sociales y están distribuidos en nuestros entornos.

Ahora bien,

\section{¿Cuáles son las metas en los procesos de aprendizaje profesional?}

Profundizar sobre los aprendizajes que un adulto realiza en un contexto de formación, demanda tener clarificadas las premisas anteriores sobre los procesos de aprendizaje o procesos de cognición en sentido amplio, para avanzar hacia procesos de aprendizajes más específicos que se dan en una carrera para profesionales de la salud, por ejemplo.

Al plantearnos como pregunta cuáles son las metas en el aprendizaje profesional de un campo, nos planteamos en otras palabras, en vistas a qué o a quién ayudamos a aprender a los estudiantes que se forman en nuestras carreras.

La respuesta parece clara a nivel declarativo: en vistas a un profesional experto. Pretendemos que los procesos de aprendizaje de nuestros estudiantes sean lo suficientemente profundos para que, desde el primer año de la formación de grado, permitan la construcción de competencias complejas que se vayan asemejando con el tiempo a la "expertise" del médico, del licenciado en enfermería, del licenciado en kinesiología, entre otros profesionales del campo.

Llegados a este punto, y recuperando las premisas sobre el aprendizaje, podemos afirmar que: se aprende a ser un profesional experto, quiere decir que tenemos posibilidades de construir individual y socialmente, de manera contextualizada y a través de "otros" (objetos y sujetos) distribuidos en dichos entornos, determinadas competencias complejas específicas que nos cualifican para una tarea profesional. No se "nace" experto, se construye la "expertise" a través de muchos años de formación y práctica, donde los primeros 5 años, son "sólo" (¿?) el punto de partida de ese camino.

Si compartimos que se aprende a ser experto, nos parece importante reflexionar sobre los procesos cognitivos que realizan los expertos, tanto en las dimensiones declarativas como las de la praxis, es decir, sobre aspectos intelectuales-conceptuales y de procedimientos y comportamientos. 
Según las investigaciones en la materia el conocimiento experto, como todo conocimiento, se construye y se le puede atribuir similares características a las abordadas en la primera parte de este escrito, sin embargo, la diferencia sustancial pareciera estar en los modos de organización que va presentando este conocimiento construido.

Dichos modos de organización podrían ser análogos a redes cognitivas altamente significativas, con patrones de reconocimiento que le permiten al sujeto disponer de conocimientos y comportamientos complejos de manera casi automática, para la resolución de problemas pertinentes a esas redes. Un experto tendría la cualidad de resolver los problemas de su práctica de manera efectiva y veloz, activando patrones de recuperación de la información o de vinculación de informaciones no relacionadas con anterioridad.

En este punto, quisiéramos acentuar las cualidades de estas redes cognitivas y la capacidad de recuperación y vinculación de lo necesario para resolver de manera experta un problema, ya que el nudo crítico estaría en las características de estas redes y en las posibilidades de acceso que habilitan.

Las mismas se construirían sobre las bases de unidades de conocimiento (conceptuales, procedimentales y valorales) significativas, profundas, de cimientos sólidos vinculados a conocimientos previos adecuados (no erróneos).

Durante los procesos de aprendizaje profesional se estarían construyendo las bases de conocimientos sólidos que necesitan tiempo y adecuación. A la par de dichos procesos de aprendizaje vinculados a los contenidos, el desarrollo de habilidades de meta-cognición son fundamentales para la construcción de futuros de mecanismos de reconocimiento de los problemas. Desde los inicios se tendría que empezar a controlar el propio pensamiento sobre lo aprendido, generar procesos de auto-explicación y comprensión permanentes, y de reconocimiento de las propias limitaciones o áreas de desconocimiento.

En relación específica con el campo de la salud, se viene estudiando sobre las características del razonamiento clínico del novato al experto y de las etapas que van desde:

1. redes fundamentalmente causales y de consecuencias de las enfermedades,

2. siguiendo por las subsiguientes compilaciones de dichas redes causales, que a medida que las redes se multiplican y la experiencia reflexionada se desarrolla,

3. se evoluciona hasta la etapa de modelos mentales propios (scripts), sustentados en las propias experiencias y representaciones del mundo, bien organizados y con acceso permanente para su recuperación casi automática.

Estas redes que en un principio se encuentran más encapsuladas, a medida que se complejizan van entretejiendo conexiones que facilitan fundamentalmente el acceso a información o las posibilidades de su gestión, para la resolución efectiva de los problemas.

Es así que desde esta mirada hacia el conocimiento experto y su aprendizaje es que reflexionamos sobre la cualidad dinámica que tiene el aprendizaje y su estructuración hacia sistemas de redes cognitivas que no dejan de evolucionar con el tiempo. 
Aprendemos re-construyendo la realidad y lo hacemos de manera dinámica y con posibilidades de evolución cualitativa de aquello aprendido. $Y$ en este sentido, las propuestas de formación profesional deberían contemplar estos procesos para ayudar a desarrollar redes de conocimientos y comportamientos pasibles de organizarse de manera significativa para su recuperación ante situaciones problemáticas de complejidad creciente en el transcurso de la formación. Potenciando la construcción de la expertise individual en un novato en formación a partir del intercambio con los expertos de la profesión y expertos en la enseñanza de la misma.

En síntesis, reflexionar y entender nuestros procesos de aprendizaje profesional, nuestro propio caminar desde novatos, nos podría ayudar como profesores de las asignaturas a diseñar mejores propuestas de formación profesional que posibiliten la generación de redes de conocimiento que recuperan de modo permanente los aspectos conceptuales, prácticos y actitudinales propios de nuestras profesiones.

Patricia B. Demuth

Campus Virtual Facultad de Medicina de la UNNE 